\title{
EL RETO DE CUIDAR EN UN MUNDO GLOBALIZADO ${ }^{1}$ THE CHALLENGE OF CARING IN A GLOBALIZED WORLD O DESAFIO DE CUIDAR EM UM MUNDO GLOBALIZADO
}

\author{
Silvina Malvárez
}

\begin{abstract}
${ }^{1}$ Conferencia Inaugural del 6to Congreso Nacional de la Asociación de Enfermería Comunitaria y 1er Simposio Internacional de Enfermería Comunitaria. Valencia, España, Noviembre de 2006. Actualizado Julio de 2007.

${ }^{2}$ Licenciada en Enfermería. Magister en Salud Materno-Infantil. Especialista en Políticas y Administración en Salud Mental. Doctora en Ciencias de la Salud. Asesora Regional de Recursos Humanos de Enfermería y Técnicos en Salud de la Organización Panamericana de la Salud. Oficina Regional de la Organización Mundial de la Salud.
\end{abstract}

PALABRASCLAVE: Atención de enfermería. Política de salud. Salud mundial. Ciencia, tecnología y sociedad.

KEYWORDS: Nursing care. Health policy. World health. Science, tecnology and society.

PALAVRAS - C HAVE: Cuidados de enfermagem. Política de saúde. Saúde mundial. Ciência, tecnologia e sociedade.
RESUMEN: El artículo aborda una reflexión sobre las principales tendencias de la humanidad en el mundo globalizado. Se define y caracteriza la globalización y sus consecuencias sociales y ambientales, entre las cuales se destaca la inequidad y el descuido como importantes impactos en la sociedad planetaria. Se indican los principales efectos en la salud y las oportunidades que abre para ella la globalización. Se analiza el papel del cuidado en la existencia humana y su condición de objeto de estudio e intervención de la enfermería. Las tendencias del desarrollo de la enfermería en los últimos años son caracterizadas en un doble proceso de cambio. Se puntualizan las implicancias de la globalización para la transformación del cuidado de enfermería y se mencionan los nuevos desafíos para el futuro, proponiéndose a la enfermería como el arte, la ciencia, la filosofía, la ética y la política del cuidado humano.

ABSTRACT: This paper presents a reflection on the main tendencies of humanity in the globalized world. This study analyzes globalization and its social and environmental consequences, highlighting inequity and the lack of care as principal impacts upon the planetary society. We indicate their effects on health care and the opportunities that globalization opens for them. The role of care in human existence is analyzed and its condition as the subject of study and intervention in the field of nursing. The developmental tendencies in the nursing profession are characterized in a double process of exchange. Implications of globalization for the transformation of nursing care and new challenges for the future are pointed out, proposing nursing as art, science, philosophy, ethics and policy of human care.

RESUMO: O trabalho aborda uma reflexão sobre as principais tendências da humanidade no mundo globalizado. Nele, se define e se caracteriza a globalização e suas conseqüências sociais e ambientais, entre as quais se destaca a iniqüidade e a falta de cuidado como importantes impactos na sociedade planetária. São indicados os principais efeitos na saúde e as oportunidades que a globalização abre para ela. É analisado também o papel do cuidado na existência humana e sua condição de objeto de estudo e intervenção da enfermagem. As tendências do desenvolvimento da enfermagem nos últimos anos são caracterizadas por um duplo processo de mudanças. Por fim, se analisam as implicações da globalização para a transformação do cuidado de enfermagem e se mencionam os novos desafios para o futuro, propondo-se a enfermagem como o arte, a ciência, a filosofia, a ética e a política do cuidado humano.
Endereço: Silvina Malvárez

2450 Virginia Ave (NW) Apt \# E615

20037 Washington DC - USA

E-mail: smalvarez@covad.net
Reflexão teórica

Recebido em: 15 de fevereiro de 2007 Aprovação final em: 23 de julho de 2007 


\section{INTRODUCCIÓN}

La transición de era se desenvuelve en una densa trama de procesos sociales de diferentes categorías y dinámicas: los procesos históricos y culturales parecen haber ingresado en un tiempo de aceleración de la historia y de transculturación consecuente a la transición demográfica. Los cambios geopolíticos devenidos de la globalización de la economía establecieron un nuevo orden internacional de liberalización de los mercados a escala planetaria. La explosión científico tecnológica ha producido avances sin precedentes en la mejora de la calidad de vida, instalando el conocimiento y la tecnología de la información como insumo estratégico principal del desarrollo y abriendo nuevos escenarios para el desempeño laboral. La transformación comunicacional vincula con increíble velocidad a las personas y a las instituciones e instala nuevas lógicas de funcionamiento global. Las dificultades de funcionamiento se producen simultáneamente en las instituciones responsables de la cohesión social, en las relaciones entre economía y sociedad y en los modos a través de los cuales se forman las identidades individuales y colectivas. ${ }^{1}$

Pero la globalización no es un proceso lineal. Presenta contradicciones y conflictividad crecientes que impactan en la salud de las poblaciones a escala planetaria. Ha sucedido mejoramiento de la vida cotidiana y de las condiciones de salud en el mundo. La tecnología de la información ha impactado radicalmente en los servicios de salud. Pero aumentaron las desigualdades, la pobreza, la marginalidad, el ataque al medio ambiente y la enfermedad influidas por el grado de inequidad en la distribución de los ingresos, mientras que los indicadores de salud dan cuentas de que las sociedades más sanas no son necesariamente las más adineradas.

En el año 2000 la Cumbre del Milenio propuso un esfuerzo planetario para abordar las prioridades mas acuciantes de la humanidad. Las Metas de Desarrollo del Milenio indican que es imperativo erradicar la pobreza extrema y el hambre, lograr la enseñanza primaria universal, promover la igualdad de género y la autonomía de la mujer, reducir la mortalidad infantil, mejorar la salud materna, combatir el Síndrome de Inmunodeficiencia Adquirida (SIDA) y otras enfermedades, garantizar la sustentabilidad del medio ambiente y fomentar una alianza mundial para el desarrollo.

En el contexto de estos cambios mundiales el descuido, parece convertirse en una de las consecuen- cias más dramáticas de la globalización, mostrando una suerte de crisis civilizatoria generalizada que se expresa justamente en el abandono de niños y ancianos, en la soledad de los adolescentes, en el abandono de los pobres y excluidos... en fin, en el abandono del sueño de la generosidad y la solidaridad, en la concentración individual y el abandono de la cosa pública, en fin... parecen estos, tiempos de impiedad. ${ }^{2}$

Por eso, el cuidado de la comunidad en un mundo globalizado representa un verdadero reto. Uds. han pensado bien: la enfermería, cuya razón social es el cuidado -condición esencial de lo humano - enfrenta el desafío, al mismo tiempo que el imperativo ético, de un cambio de pensamiento, de posición y acción para hacer frente a las necesidades de cuidado de las comunidades en un contexto cambiante de amenazas y oportunidades.

Esta presentación hace un énfasis particular en las tendencias mundiales y el impacto de la globalización en la salud de las comunidades en la convicción que es sólo entendiendo el contexto cómo la enfermería puede ejercer mejor su misión de cuidado. Y concluye intentando reflexionar sobre los desafíos que trae para la enfermería este estado del mundo, desafíos que imponen una reflexión y una acción política profundas de modo de poder re-situar la enfermería comunitaria como el corazón mismo de esta praxis y como el campo de conocimientos, de responsabilidad social y de intervención, estructurante, dinamizador y rector de la enfermería toda.

Mi vida profesional se constituyó en el marco de la salud pública. Las experiencias de trabajo comunitario durante muchos años y en el área de la salud mental determinaron que esa fuera la única y exclusiva concepción posible que pude forjar de la enfermería, de modo que, aún trabajando en grandes hospitales psiquiátricos, jamás pude imaginarme los límites del hospital siendo el mismo siempre y necesariamente un espacio comunitario, un ámbito donde pensar epidemiológica y socialmente, un ambiente de práctica social enraizado y viviente de la comunidad misma.

\section{GRANDES TENDENCIAS}

El Informe sobre el Estado del Futuro 2005, de la Universidad de las Naciones Unidas comienza afirmando que el sorprendente desborde de ayuda humanitaria a las víctimas del tsunami parece establecer una nueva marca en la evolución ética de la humanidad. Este hecho inspira confianza en que tendremos 
la voluntad necesaria para enfrentar decisivamente los desafíos globales y ganar la competencia entre la constante proliferación de amenazas y nuestra creciente habilidad para mejorar la condición humana. ${ }^{3}$

La población mundial ha llegado a los 6.500 millones. Las futuras sinergias entre nanotecnología, biotecnología, tecnologías informáticas y ciencias del conocimiento pueden mejorar drásticamente la condición humana por el crecimiento en la disponibilidad de alimentos, agua y energía y por el mayor intercambio de información entre las personas de todas partes. La consecuencia será el aumento de la inteligencia colectiva y la creación de mayor valor y eficiencia junto a la reducción de costos.

Sin embargo, aunque la humanidad dispone de los recursos suficientes y pertinentes para enfrentar los desafíos globales, no se vislumbra aún, cuánta sabiduría, voluntad e inteligencia se dedicarán a los mismos.

Las tendencias demográficas muestran que la población mundial crece a un ritmo de $1,2 \%$, sin embargo, no son parejas: la población aumentará considerablemente en los países en desarrollo y tenderá a reducirse en los desarrollados.

Para fines del 2007, la mitad de la población mundial vivirá en zonas urbanas en ciudades de no más de 500.000 habitantes, aunque también aumentan los grandes conglomerados urbanos: Tokio, México, Bombay, New York, São Paulo y Delhi reúnen más de 15 millones de personas cada uno. ${ }^{4}$ Muchas de estas ciudades tienen enormes poblaciones de pobres, analfabetos, desempleados, niños en la calle, jóvenes violentos y ancianos abandonados, como efecto de la urbanización descontrolada. Las tendencias poblacionales muestran el gran aumento de flujos migratorios que se concentran en ciudades. El envejecimiento poblacional es una tendencia creciente. Las tasas de fecundidad deficitarias o de reemplazo se dan en países desarrollados, mientras que aumentan en países en desarrollo.

En el siglo XX se produjo el más rápido descenso de la mortalidad general registrado en la historia de la humanidad gracias a las mejoras en las condiciones de vida, aunque el SIDA está produciendo aumentos en la mortalidad en África. Existen diferencias demográficas entre los países en desarrollo y los desarrollados: los primeros mantienen altas tasas de mortalidad materna, infantil y por $\mathrm{VIH}$, mientras que en los segundos el envejecimiento poblacional y el descenso de la fecundidad preocupan a los países.
Las tendencias de cambio social muestran creciente capacidad de cooperación y mejoramiento de la calidad de vida de algunos pueblos, junto con intranquilidad política y social, estrés, aumento del fundamentalismo, el terrorismo y choques entre ricos y pobres.

Las tendencias ambientales exhiben el incremento de desastres naturales al mismo tiempo que una voluntad general hacia el desarrollo sustentable.

Las tecnologías de la información han producido enormes avances en las ciencias y las comunicaciones, han generado redes internacionales e impactado de manera importante la fluidez en la traducción de lenguajes hacia una comunicación global.

El explosivo desarrollo de la ciencia ha permitido un enorme mejoramiento de la vida de muchas personas, al mismo tiempo que generado tecnologías cuyo uso discrecional amenaza el ambiente y la seguridad de las naciones. La tecnología del transporte ha facilitado los flujos migratorios y el incremento a escala planetaria de las transacciones comerciales.

Pero, en este marco, la más impactante tendencia es la de la globalización, marco determinante y condicionante del nuevo orden mundial.

\section{GLOBALIZACIÓN}

La globalización, es un proceso económico, social y cultural establecido en las dos o tres últimas décadas del siglo XX, cuyas principales características incluyen, en escala nunca antes alcanzada, ${ }^{5}$ las siguientes:

- crecimiento del comercio internacional de bienes, productos y servicios;

- transnacionalización de mega-empresas;

- libre circulación de capitales y competivividad económica basadas en el uso intensivo del conocimiento;

- privatización de la economía y minimización del papel de los gobiernos y de los Estados-Nación;

- fortalecimiento de barreras comerciales proteccionistas y regulación del comercio internacional según las reglas de la OMC;

- facilidad de tránsito de las personas y los bienes entre los países;

- expansión de las posibilidades de comunicación, surgimiento de la sociedad de la información e incremento del contacto entre las personas. ${ }^{5}$ 
La globalización es la interconectividad del capital, la producción, las ideas y la vida cultural en marcha constante y creciente, mientras que, implícita en la idea de globalización mas que internacionalización, yace la idea de que nos movemos hacia una era de crecientes lazos entre naciones que superan la noción del estado-nación, trayendo consigo un cambio profundo en la concepción espacio-tiempo y en la dimensión congnitiva, flujos globales crecientes y aumento y re-configuración de actores y escenarios. La globalización instala un capitalismo de progreso unilateral concentrador de riqueza y poder, lo cual elimina las expectativas de una globalización con beneficios para todos. El efecto redistributivo resulta, en verdad, inverso.

La globalización ha determinado que para muchos la vida sea hoy más larga y saludable que en cualquier otra época de nuestra historia. La esperanza de vida al nacer aumentó en los últimos 40 años más rápidamente que en los 4000 años que la precedieron. Pero no todos se beneficiaron por igual; en la mayoría de los países existen marcadas diferencias.

Muchos autores y organizaciones son críticos de este proceso. La Comisión Mundial sobre Dimensiones Sociales de la Globalización insiste en que el proceso de globalización actual está produciendo resultados desiguales entre los países y al interior de los mismos y son demasiadas las personas que no participan de los beneficios.

Así, que los mercados globales son inherentemente "desigualizantes" y provocan el incremento de desigualdad entre los países y aún dentro de ellos. La enorme diferencia del promedio de ingresos entre los países más ricos y los más pobres ha crecido de 9 a 1 en el 1900 a 100 a 1 hoy en día y los países más ricos hoy - que ya lo eran hace 100 años como los de Europa, Norte América y Australia, continúan enriqueciéndose. ${ }^{6}$

Aún cuando la riqueza mundial actual estimada en 24 trillones de dólares anuales - sigue aumentando, cerca de 1,2 billones de personas en el mundo viven con menos de 1 dólar por día y la mitad de los habitantes del mundo viven con menos de 2. En Asia meridional el 37\% es pobre (448 millones de personas). En América Latina y el Caribe el número de personas pobres creció de 48 a 57 millones durante la década del '90: una de cada 10 personas en la región vivía con menos de un dólar al día cuando comenzaba el milenio.?
La Organización Internacional del Trabajo (OIT) afirma que las desigualdades globales son inaceptables desde el punto de vista moral e insostenibles desde el punto de vista político. Estas condiciones constituyen la base material para el impulso de cambios de valores, de ética y de comportamientos de los individuos, de los estados, instituciones y organizaciones. ${ }^{8}$

\section{Inequidades en salud}

Una de las principales marcas de la globalización radica en el modo como sus mecanismos se expresan, producen, sostienen e incrementan la inequidad en salud constituyendo un estado de injusticia social.

En los países más desarrollados del mundo la esperanza de vida al nacer era en el 2001 de 78 años y de 51 en los menos desarrollados; la mortalidad infantil de niños menores de 1 año es de 6 por 1000 nacidos vivos en los primeros y 100 por 1000 en los segundos, mientras que en la de menos de cinco asciende a 159 en los países más pobres.

En Sudáfrica la mortalidad infantil es cinco veces mayor entre los negros que entre los blancos; los varones que viven en los condados más ricos de Estados Unidos pueden esperar 16 años más de vida que los de los más pobres; en Chile las personas menos educadas son las que más enferman; en Inglaterra las mujeres con afecciones crónicas tienen más posibilidad de perder su empleo que en Suecia; en Japón, los trabajadores agrarios tienen menos esperanza de vida que los gerentes y profesionales. ${ }^{9}$

En América Latina la mortalidad infantil disminuye en estado constante. Pero las desigualdades son gigantes: mientras en Bolivia mueren 54 niños de cada 1000, solo 5,3 mueren en Canadá. ${ }^{10}$ Más de 1000 bebés menores de un año mueren cada día en las Américas; cada año 140.000 niños mueren a causa de enfermedades prevenibles antes de los cinco años. ${ }^{7}$

Hubo cambios pocos significativos en la evolución de la mortalidad materna siendo el riesgo de muerte enormemente más alto en el país más desarrollado que en el menos desarrollado; cada 25 minutos una mujer muere en Latinoamérica y el Caribe por causes relacionadas con el embarazo, siendo el riesgo 28 veces superior a Norteamérica.

En Brasil la mortalidad infantil entre los negros es de 34 por 1000 contra 23 entre los blancos; entre los pobres es de 35 y 16 entre los ricos; entre las madres con menos de 3 años de estudio es de 40 
contra 17 entre las madres con 8 años de estudios o más; en la población rural es de 35 contra 27 en la urbana; en el estado más pobre es de 63 por 1000 contra 16 en el más rico. ${ }^{5}$

Las diferencias en el uso de los servicios de salud también se observan entre países y jurisdicciones y entre ricos y pobres, en donde las diferencias varían de 1,3 a 4,8 veces en relación al uso de terapias de rehidratación, anticonceptivos, consultas prenatales y atención calificada del parto. Las inequidades a través de Europa persisten; las diferencias de acceso a los servicios de salud entre las distintas clases sociales y segmentos de la población continúan y aún crecen. ${ }^{11}$

El gasto per cápita en salud varía de 11 dólares anuales en los países menos desarrollados del planeta a 1.907 en los países más desarrollados.

En resumen, la globalización ha empobrecido a muchos países y ampliado la exclusión y las inequidades, las cuales, en la mayoría son injustas y evitables. Las desigualdades se deben a diferencias en el acceso a renta estable, recursos sociales, trabajo, asistencia, protección social, agua, servicios sanitarios y, lo que es más importante, a educación factor determinante de las condiciones de salud.

La globalización trae también consigo consecuencias sobre condiciones y enfermedades específicas, por ejemplo las derivadas del turismo sexual internacional y la transnacionalización de las enfermedades transmisibles nuevas y re-emergentes; el Síndrome Respiratorio Agudo Severo (SARS), el dengue, la gripe aviar, las fiebres hemorrágicas virales, la salmonelosis y la escherichia-coli son algunos ejemplos de ello. Entre las llamadas enfermedades re-emergentes se cuentan la poliomielitis que abunda en 15 países de África por déficit de vacunación, la epidemia de cólera que afectó a 75 países en los últimos 40 años, la fiebre amarilla y la tuberculosis resistente, entre otras. ${ }^{7}$

En el 2001, 40 millones de personas vivían con SIDA en el mundo y de ellos 3 millones morían por año. La proyección indica que para el 2020 morirán 68 millones de personas en los 45 países más afectados. En Latinoamérica y el Caribe, cerca de 119.000 personas murieron a causa del sida en 2003 y unas 200.000 resultaron infectadas con el VIH. Cerca de 1.000.000 de personas en la región sufren de malaria.?

El siglo XX ha sido uno de los períodos más violentos de la historia de la humanidad: 191 millones de personas perdieron la vida a causa de conflictos. $\mathrm{La}$ violencia y la criminalidad se ha incrementado en las grandes ciudades en un $40 \%$ en la década de los 90 , los disturbios civiles y las guerras dejan como consecuencia más muertes, lesiones y discapacidad. ${ }^{7}$

Las nuevas sociedades traen consigo padecimientos crónicos que convocan la atención de las comunidades y del sector sanitario en particular. Las enfermedades cardiovasculares, las neoplasias, la diabetes y la hipertensión constituyen las primeras causes de morbimortalidad en los adultos de países desarrollados; los desordenes en la nutrición ofrecen un cuadro de tendencias bidireccionales: la desnutrición aumenta en los niños y afecta a muchas madres de los países pobres y la obesidad, se incrementa hasta alcanzar a casi más de la mitad de la población de algunos países ricos.

Un grupo de padecimientos sistemáticamente descuidado ha sido el del sufrimiento psíquico y los trastornos mentales y del comportamiento. Estudios epidemiológicos mundiales indican que 400 millones de personas en el mundo sufren de trastornos mentales severos y que integran el $8 \%$ de la carga mundial de enfermedad; el $25 \%$ de la población de las Américas padece actualmente algún tipo de estos trastornos y para el año 2010 se espera que una de cada tres personas sufra de depresión; hay un alto índice de suicidio, un aumento de las adicciones a substancias psicoactivas, al alcohol y al tabaco. ${ }^{7}$ A pesar de los esfuerzos realizados para mejorar la atención de la salud mental, el estigma, la desatención y la violación a los derechos humanos de personas con sufrimiento psíquico completan este dramático cuadro.

Los desastres naturales crecientes debido al ataque al ecosistema planetario, ocasionan muertes y lesiones múltiples, pérdidas de vivienda, desprotección, inseguridad, desesperanza, desplazamientos, mayor pobreza y consecuentemente más enfermedad. La migración forzada y la globalización del tráfico de drogas completan el cuadro de la salud en la globalización. En Latinoamérica y el Caribe, 130 millones de personas ( 1 de cada 4$)$ no tiene acceso a agua potable en casa y menos de 1 de cada 5 está conectada a sistemas de saneamiento adecuado. ${ }^{7}$

La reforma del sector salud, por su parte, supuso implementación de nuevas políticas, cambio en las estructuras y organización de ministerios y servicios, cambios en el financiamiento y las reglamentaciones, retiro del Estado de la responsabilidad de rectoría de la salud y mayor participación del sector privado en los servicios. En muchas naciones aumentaron las inequidades y la desigualdad de ac- 
ceso a la atención existiendo en la actualidad, por ejemplo en América Latina, más de 200 millones de personas que carecen de protección social y $100 \mathrm{mi}$ llones sin acceso a los servicios básicos de salud.

Los recursos humanos en salud, componente activo más importante del sector, exhiben viejos y nuevos problemas. Su cantidad y distribución son inadecuadas, las migraciones amenazan sistemas de salud en países pequeños, las desvinculación entre educación, servicio y prioridades de salud produce frustraciones en los profesionales e ineficiencia en la atención; las condiciones de trabajo se precarizaron en los países menos desarrollados y las prácticas tradicionales colisionan con los requerimientos del sistema. Sin embargo, el estatus político de los crisis de recursos humanos en salud ha ascendido a la agenda internacional como factor crítico para la salud, la economía y la seguridad mundial. En función de ello, una alianza mundial a favor del desarrollo de recursos humanos en salud planteó en 2006 un plan de desarrollo de largo plazo para contribuir al logro de los Objetivos del Milenio.

\section{Oportunidades}

Con todo, la globalización trae también señales positivas. ${ }^{5}$ La creación del sistema de Naciones Unidas a mediados del siglo XX y de la Organización Mundial de la Salud (OMS), representó un avance importante para el diálogo y la convivencia pacífica de las naciones así como para la cooperación a favor del progreso de todos los países y personas del mundo.

En la última década del siglo XX se instaló un sistema de diálogo internacional caracterizado por el desarrollo de grandes conferencias temáticas con miras a preparar el mundo para el siglo venidero. Más de 15 conferencias y cumbres mundiales debatieron y emitieron declaraciones en favor de los niños, el medio ambiente, los derechos humanos, las poblaciones, la mujer, el desarrollo social, la alimentación, el desarrollo sustentable, el financiamiento y los objetivos del milenio, y se conformaron comisiones mundiales de estudios sobre macro-economía y salud, sobre determinantes sociales de la salud, entre otros

La salud se ha instalado en la agenda política mundial como una cuestión de seguridad, de política exterior, de macro-economía y de derecho humano y de estas condiciones ha emergido el concepto de salud global como un nuevo contexto, una nueva conciencia y una nueva estrategia de abordaje del gobierno de la salud en la sociedad planetaria. Esta intencionalidad colectiva constituye un esfuerzo global en pos de la construcción de una nueva agenda: la de aprender a ser una sociedad mundial. ${ }^{8}$

La globalización también puede concebirse como una fuerza emancipadora, provista de la información y la capacidad para construir puentes y utopías. Los progresos de la comunicación y la información permiten que el mundo esté más cerca de cada uno; no importa donde, la humanidad parece más próxima, la conciencia de ser mundo se instala en los lugares por la presencia de una humanidad mezclada de razas, culturas, lenguas, renovado entendimiento y tolerancia, enriqueciendo el cotidiano individual y colectivo. La mutación tecnológica, a partir de la ingeniería genética, autorizó la promesa de la mutación biológica del hombre; pero una mutación filosófica está esperando, la del hombre capaz de atribuir un nuevo sentido a la existencia de la persona, de la humanidad y del planeta. ${ }^{12}$

\section{A cerca del cuidado}

Leonardo Boff, ${ }^{2}$ teólogo y filósofo brasilero, Premio Nobel Alternativo de la Paz, miembro de la Comisión de la Carta de la Tierra, dice en su libro "Saber cuidar" que la crisis generalizada que afecta a la humanidad se revela por el descuido con que se tratan las realidades más importantes de la vida. La crisis es civilizatoria. Para salir de ella se precisa una nueva ética que ha de nacer de algo esencial en el ser humano. La esencia humana reside mucho más en el cuidado que en la razón o en la voluntad. Cuidar es más que un acto, una actitud. ${ }^{2}$

El más grande estudioso del cuidado, Martín Heidegger, ${ }^{13}$ en su famosa obra Ser y Tiempo entiende que el cuidado, desde el punto de vista existencial, es ontológicamente anterior a toda actitud o situación del ser humano. El cuidado se encuentra en la raíz primera del ser humano y representa un modo-de-ser esencial, presente, irreductible, constituyente y base posibilitadota de la existencia humana. Habla del cuidado como anticipación, ocupación y solicitud, entendiendo que la noción de alteridad es intrínseca al mismo y que la expresión "cuidado de sí" sería una tautología. ${ }^{13}$

El cuidado surge cuando la existencia de otro adquiere importancia para mí, en consecuencia, me dispongo a participar de su existencia; es ese modo-de-ser mediante el cual salgo de mí para 
centrarme en el otro con desvelo y solicitud, desplazo la preocupación por mí misma y hago que el otro tome prevalencia.

El cuidado incluye dos significados íntimamente ligados: el primero, la actitud de desvelo, de solicitud, de atención hacia el otro; el segundo, la preocupación, la inquietud, la involucración, porque supone una implicación afectiva con el otro cuya condición conmueve mi actitud y moviliza mi acción. Pero cuidar también es pensar; cuidar proviene de cogitare que significa pensar este segundo significado de preocupación e inquietud se vincula con el concepto de cuidado como pensamiento en el otro.

La historia del cuidado humano es tan extensa como la historia de la humanidad, sin embargo, el proceso de profesionalización del cuidado se liga a la historia de la atención de la enfermedad, más específicamente al desarrollo de las instituciones de atención médica y al de la medicina como ciencia.

La historia le ha dado a la enfermería el incomparable encargo social del cuidado profesional. No entender estas raíces y la trascendencia de este encargo, significa no entender la misión social de la enfermería como profesión.

La evolución histórica de la enfermería y su articulación orgánica con los procesos sociales, políticos y científicos, ha confluido en un estatus presente de configuración profesional que la ubica en un lugar destacado de aporte al bienestar humano y una condición de imprescindibilidad de su misión en la prevención de las enfermedades y la recuperación de la salud; pero lo que es más importante, un reconocimiento de su contribución a la atención de las personas enfermas o en riesgo, no para curar, sino para confortar, para hacerse cargo del impacto de la enfermedad en la persona total, para complementar las capacidades debilitadas y potenciar las presentes, para aliviar, para asegurar.

Los últimos 30 años han representado para la enfermería un proceso de despegue que pocos hubieran imaginado tiempo atrás, proceso en que la enfermería comienza a constituirse como actor social y, en una ética consistente con ese pensamiento, produce análisis inteligentes, desencadena programas estratégicos y crea condiciones políticas, técnicas y humanas para conducir el desarrollo. Ese nuevo lugar es, en realidad, un lugar de distinción para el cuidado humano, el lugar que las sociedades y los sujetos, que saben de su trascendencia para la vida, le otorgan con carácter de imprescindible.

Por eso es posible pensar que el contexto global, la enfermería transita un doble proceso de cambio: un cambio regresivo y otro progresivo que permite advertir positividades y negatividades, unidad en la diversidad, dialogicidad y movimiento.

El cambio regresivo ajusta la enfermería de muchos países, en especial los países en desarrollo a las consecuencias negativas de la globalización, las diversidades e inequidades del desarrollo y las consecuencias de la reforma del sector salud.

En contraste, un cambio progresivo también esta sucediendo, como expresión del cual la contribución de las enfermeras a la salud se registra en la valoración mundial como imprescindible; el progreso en el conocimiento, el uso de la tecnología, el proceso de profesionalización y la participación de las enfermeras en las decisiones de políticas de salud, representan, entre otras, señales de ese progreso.

Tampoco es posible caracterizar un mundo igual en la enfermería. Las diferencias, desigualdades e inequidades de la globalización también la incluyen conformando un mapa multicolor en el marco del cual, con todo, emerge la unidad de la esperanza: dondequiera que uno se encuentre, cualquiera sea la enfermera con la que se dialogue, la esperanza, la voluntad y el amor a la gente, emergen como una distinción promisoria y unívoca: la de un mejor cuidado para la humanidad.

\section{El reto de cuidar}

Las transiciones demográfica y epidemiológica de la humanidad unidas a las consecuencias de la globalización presentan un panorama cambiante que exige de la enfermería un franco re-posicionamiento en relación a su tradición profesional. La noción de salud global no es un mero concepto, es también la vivencia de mundo de verificación cotidiana y una realidad creciente que demanda pensamiento y responsabilidad por la humanidad planetaria.

El envejecimiento poblacional, la urbanización, la disminución de la fecundidad, las migraciones y las causas diversas de la mortalidad determinan que las enfermeras debam rápidamente prever estrategias de educación y planificación para asegurar cuidados pertinentes a los ancianos, a las

Malvárez S. La investigación en enfermería en el contexto de la salud mundial. Conferencia dictada en el VIII Coloquio Panamericano de Enfermería. México, 2002. 
víctimas de la violencia urbana, a los problemas de transculturación de poblaciones completas y a las nuevas causas de la mortalidad.

Los desastres crecientes y los conflictos bélicos demandan de la enfermería el urgente desarrollo de competencias para proveer cuidados en situaciones de emergencia y programar cuidados para enfrentar las consecuencias mediatas de esas situaciones, incluida la discapacidad.

La pobreza, el analfabetismo, la desnutrición, la desprotección social, exigen de las enfermeras una revisión de su misión y una reorientación franca hacia la consideración de los procesos sociales micro y macro determinantes de la salud y la enfermedad.

La mortalidad materna y la mortalidad infantil por causas prevenibles constituyen un foco central de la responsabilidad de las enfermeras.

Las enfermedades infecto-contagiosas, su capacidad de diseminación a nivel planetario y su amenaza con la destrucción de comunidades completas, requieren de las enfermeras un fortalecimiento y re-direccionamiento epidemiológico de sus bases conceptuales, de sus intervenciones y de su capacidad anticipatoria y preventiva. Se requiere que el abordaje del SIDA, como cuestión global, ingrese definitivamente en la preocupación y en la agenda de cuidados integrales de la enfermería.

El suicidio, las drogas y los problemas de sufrimiento psíquico requieren adquirir primacía en la preocupación y el desarrollo de las enfermeras.

El problema de la inequidad de acceso a las riquezas, a la atención de salud y a la información debe constituir el centro crítico de una nueva visión de la enfermería. La inequidad subyace a la gran mayoría de los problemas de salud, constituye una cuestión política y supone renovadas intervenciones sociales basadas en una ética del derecho humano a la salud, a la libertad y a la ciudadanía. Asumir el problema de la inequidad supondrá para la enfermería enormes cambios: implicará hacer conciente la propensión a la inequidad, reconocer que la inequidad se expresa en la salud de manera dramática, influir en el cambio de los sistemas de salud hacia un cuidado de acceso universal y generar prácticas óptimas, pero el cambio esencial será el de asumirse como sujeto político en lucha por una humanidad más justa.

La inequidad es también un mal de la enfermería. Basta con una mirada rápida a los salarios, las jornadas de trabajo, los recursos materiales, el acceso a la formación y el reconocimiento social de las enfermeras, para tomar dolorosa conciencia de esas desigualdades.

Tomar en cuenta la crisis de los recursos humanos en salud en el mundo, constituye un imperativo de salud global de difícil pero imprescindible y urgente abordaje. Supone poner el foco en las prioridades de salud de las naciones y en las condiciones de acceso a la atención. Luego requiere un esfuerzo consciente para identificar insuficiencias, distribución inequitativa, falta de competencias, desmotivación, malas condiciones de trabajo, motivos de abandono y causas de la movilidad internacional, con el objeto de delinear la magnitud de la crisis. Exige una nueva comprensión de los recursos humanos en salud como campo de fuerzas donde educación y trabajo, mercado de trabajo, procesos de profesionalización, políticas, regulación y gestión en el contexto de la dinámica económico social, configuran un escenario complejo de actores, intereses y tensiones que demandan intervenciones a largo plazo.

La meta ha de ser el acceso equitativo para todas las poblaciones a una fuerza de trabajo adecuadamente formada, competente y motivada para contribuir a lograr el más alto nivel posible de salud. ${ }^{14}$ Esto implica un esfuerzo global de imaginación, cooperación internacional y alianzas para construir una nueva historia de equidad, pertinencia, compromiso social y bienestar de la fuerza de trabajo en salud, de la cual las enfermeras representan una importante parte.

\section{COMPRENDER EL RETO}

El reto se construye como amenaza y oportunidad. Según la Lic. Beatriz Gómez, distinguida Profesora de Ética de la Universidad Nacional de Córdoba, Argentina, fallecida hace un año, el desafío del cuidado de las comunidades en un mundo globalizado, se inicia con dos preguntas:

- ¿Estamos cuidando a todos?

- ¿Estamos cuidando bien?†

El desafío que tiene la enfermería por delante no es breve, no es simple: es el desafío de cuidar el mundo y el desafío de cuidarlo bien.

La base ético-política de este reto ante el escenario global, tiene implícitos, a mi modo de ver, seis valores esenciales: 
- el valor del derecho a la salud y a la ciudadanía;

- el valor de la equidad;

- el valor de la excelencia;

- el valor de la tolerancia;

- el valor de la solidaridad y;

- el cuidado como valor existencial.

El desafío de cuidar a las comunidades en un mundo globalizado y de cuidarlas bien supone una transformación paradigmática de la noción de cuidado de enfermería, históricamente focalizado en el individuo, que necesariamente ha de partir de la conciencia de mundo y que implica la adopción de la comunidad global como unidad de análisis y sujeto de cuidado.

El desafío de cuidar el mundo y de cuidarlo bien, supone un cambio de problemas, un cambio de abordajes, un cambio de tecnologías, escenarios diferentes y prácticas distintas.

E implica la comprensión de las dialécticas mundo-región-mi país-mi ciudad. Así, tanto la figuración del estado de la salud en el mundo cuanto nuestra preocupación y compromiso con ella, pasan al mismo tiempo por el entendimiento y cuidado de esta pequeña parte de mundo que es "mi ciudad" en donde el mundo se expresa integralmente, por el entendimiento de las intermediaciones globales que, sin metáforas, hacen de "mi ciudad", el mundo y por la comprensión, la conciencia, la preocupación, la solicitud, y la acción por el buen cuidado de enfermería en otras partes del planeta.

El desafío de cuidar el mundo y de cuidarlo bien supone acción política, abogacía, participación, cooperación y renovados estilos de gestión, modelos educativos orientados a las prioridades de salud de las naciones en sí mismas y en interconexión global, nuevos objetos de investigación, una enorme acción asociativa y la definitiva e inexcusable adopción del trabajo en equipo, así como la noción de intersectorialidad.

Este salto cualitativo es requerido a la enfermería en una época donde las condiciones están dadas: las enfermeras disponen ahora del estatus global de imprescindibilidad de la enfermería, disponen de la información y el conocimiento científico, de la conectividad, de la tecnología de la salud, de la comprensión de los macro-determi- nantes, de la posibilidad de alianzas y prácticas colaborativas, de acceso a experiencias exitosas de cuidado en otras partes del mundo y de la capacidad de solidaridad y el amor por la gente, condiciones que jamás perdieron.

Las enfermeras tienen el espacio común de la esperanza y la capacidad de la solidaridad para con otras poblaciones y otras enfermeras de diferentes partes del mundo, solidaridad que también requiere globalizarse mediante acciones políticas y técnicas de cooperación internacional ordenadas a conformar una red global de cuidados. No hay modo, ahora, cómo desentenderse de las condiciones de salud en las comunidades más postergadas.

Hemos dicho en oportunidades anteriores que entendemos a la enfermería como una profesión del campo de las ciencias sociales, cuyo objeto de estudio e intervención es el cuidado humano en cuanto implicado en la constitución, vivencia, desarrollo, protección y recuperación de la salud, que tiene como beneficiario al sujeto humano como ser cultural, a la familia, los grupos, las comunidades y la sociedad planetaria como unidades de análisis y cuidado. Entendemos al cuidado en su única condición posible que es la de alteridad, vínculo amoroso y desvelo por cada sujeto y por la humanidad. ${ }^{\ddagger}$

Entendemos a la enfermería como la ciencia, el arte, la filosofía, la ética y la política del cuidado humano, que se organiza y expresa socialmente con identidad singular, en un complejo campo de conocimientos, de intervención y de responsabilidad social propios que instituyen su identidad profesional en relación con la comunidad y las otras profesiones de salud.

La enfermería es ciencia en cuanto supone un proceso de racionalización del saber ordenado por la sistemática científica, en cuanto se fundamenta y construye como conocimiento científico singular y en cuanto reflexiona metódicamente sobre su práctica, la explora, la describe, la explica, la predice y la comprende. Por fin, en cuanto aporta el saber sobre el cuidado humano al conocimiento universal.

La enfermería es arte en cuanto creación, expresión subjetiva y ajustada a cada acto de cuidado, es arte en cuanto técnica y talento están implicados en el buen cuidado y es arte en cuanto bella y armónica se propone la experiencia estética del cuidado de cada ser humano en su contexto.

* Malvárez, S. La investigación en enfermería en el contexto de la salud mundial. Conferencia de Clausura del VIII Coloquio Panamericano de Enfermería. México DF, México, 2002. 
Pero también la enfermería es filosofía en cuanto inquisición y pregunta constantes sobre la existencia humana, sobre sus vicisitudes y trascendencia y porque indaga y reflexiona sobre los fundamentos ontológicos, éticos, teleológicos y epistemológicos del cuidado.

La enfermería es ética puesto que no hay posibilidad de concepción y práctica de la enfermería sin la asunción del cuidado como valor estructurante de la entrega, la solidaridad y la responsabilidad social. En otro sentido y basado en el anterior concepto, las enfermeras no solamente desarrollan para sí, sistemas de valores y comportamientos profesionales que regulan éticamente el acto de enfermería. La ética del cuidado no reúne sólo los valores humanos que se definen por norma para este acto, sino, el modo singular de existir, de vivir y de ser cuidado.

Y por último la enfermería es la política del cuidado humano. La naturaleza inherentemente política de la enfermería se expresa en su marco ético, conceptual y práctico en cuanto piensa en poblaciones, se ocupa de los determinantes sociales de la salud y produce intervenciones sociales orientadas a la equidad; ello representa un acto de distribución de poder, que permite, mediante una acción social deliberada, construir un mundo algo mejor, más justo, bien sea por medio de un niño sanado, de una enfermedad impedida, de una condición social redimida o de una comunidad alentada más libremente gracias al cuidado.

Por eso entendemos que las enfermeras constituyen sujetos de cultura y afecto, educados para desempeñar su misión profesional de cuidado en carácter de actores sociales que participan, a través de sus relaciones de poder, en la construcción de la historia nacional y mundial, en la estructuración y dinámica de los campos de la salud y de la ciencia y en la edificación del futuro como instancia constituyente de la identidad subjetiva y social.

Esta concepción multidimensional de la enfermería contiene el intento de provocar una discusión crítica sobre su tradición histórica articulada al modelo biomédico y concentrada en el hospital, una discusión que conduzca a entender que la enfermería en sí misma nació y está llamada a recuperarse en su predominante condición comunitaria ya que es allí donde la mayor parte de los problemas de salud se desenvuelven, se expresan, pueden impedirse, cuidarse y resolverse y porque es en la comunidad donde el ejercicio de la promoción y protección de la salud puede contribuir al desarrollo social.

La resignificación y desarrollo de la enfermería comunitaria constituye en resumen el desafío mayor si se entiende que, en el contexto de la interdisciplina y la complementariedad intersectorial, la enfermería pude dar su aporte a la renovación de la Atención Primaria de Salud como sistema político, conceptual y práctico de la respuesta social organizada a la salud de las comunidades; ámbito especial y específico donde el cuidado se desenvuelva tan íntimo como comprensivo, tan solícito como abarcador y tan complejo y pertinente como calificado, ordenado a cuidar a todos y a cuidarlos bien.

\section{REFERENCIAS}

1 Tedesco JC. Educación en la sociedad del conocimiento. Buenos Aires (AR): Fondo de Cultura Económica; 2002.

2 Boff L. Saber cuidar: ética do humano: compaixão pela terra. Rio de Janeiro (RJ): Ed. Vozes; 1999.

3 Glen J, Gordon T. Estado del futuro 2005. New York (NY/USA): American Council for the United Nations University; 2005.

4 Organización de las Naciones Unidas. Problemas demográficos y objetivos de desarrollo. New York (NY/USA): ONU; 2006.

5 Buss P. Globalización, pobreza y salud [conferencia]. In: 11o Congreso Mundial de Salud Pública; 2006 Ago 21-5; Rio de Janeiro, Brasil. Rio de Janeiro (RJ): ABRASCO; 2006. 1-13.

6 Birdsall N. The world is not flat: inequality and injustice in our global economy. Helsinsky (FI): UNU/World Institute for Development Economics Research; 2005.

7 Martínez E. Forjando un futuro más justo: en perspectivas de salud. v.9. n.2. Washington (DC/ USA): OPS/OMS; 2007.

8 Kickbusch I. Global public health: the challenge for the 21st Century [Training Program in International Health. Conference for the Closing Session]. Washington (DC/USA): PAHO/WHO; 2002.

9 Evans T, Whitehead M, Diderichsen F, Bhuiya A, Wirth M. Desafío a la falta de equidad en salud: de la ética a la acción. New York (NY/USA): Oxford University Press; 2001.

10 Organización Panamericana de la Salud. Situación de salud de las Américas: indicadores básicos de salud 2006. Washington (DC/USA): OPS/OMS; 2006.

11 Bartley M. Capability and resilience: beating the odds. London (UK): Economic and Social Research Council; 2006. 
12 Santos, M. Por una otra globalización: del pensamiento único a la conciencia universal. Río de Janeiro (RJ): Record; 2006.

13 Heidegger M. Ser y tiempo. Santiago de Chile (CL):
Ed. Universitaria Temas de Filosofía; 1997.

14 Yan J. The nursing and midwifery office at WHO and the GNWHOCC. Glasgow (UK): GNWHOCC Biennial Meeting; 2006. 